\section{Genome Sequence Resources for the Wheat Stripe Rust Pathogen (Puccinia striiformis f. sp. tritici) and the Barley Stripe Rust Pathogen (Puccinia striiformis f. sp. hordei)}

\author{
Chongjing Xia, ${ }^{1}$ Meinan Wang, ${ }^{1}$ Chuntao Yin, ${ }^{1}$ Omar E. Cornejo, ${ }^{2}$ Scot H. Hulbert, ${ }^{1}$ and Xianming \\ Chen $^{1,3, \uparrow}$ \\ ${ }^{1}$ Department of Plant Pathology, Washington State University, Pullman, WA 99164-6430, U.S.A. \\ ${ }^{2}$ School of Biological Sciences, Washington State University \\ ${ }^{3}$ Wheat Health, Genetics, and Quality Research Unit, Agriculture Research Service, U.S. Department of \\ Agriculture, Pullman, WA 99164-6430, U.S.A.
}

\begin{abstract}
Puccinia striiformis f. sp. tritici causes devastating stripe (yellow) rust on wheat and $P$. striiformis $\mathrm{f}$. $\mathrm{sp}$. hordei causes stripe rust on barley. Several $P$. striiformis f. sp. tritici genomes are available, but no $P$. striiformis f. sp. hordeigenome is available. More genomes of $P$. striiformisf. sp. triticiand $P$. striiformisf. sp. hordei are needed to understand the genome evolution and molecular mechanisms of their pathogenicity. We sequenced $P$. striiformis $\mathrm{f} . \mathrm{sp}$. tritici isolate 93-210 and P. striiformis f. sp. hordei isolate 93TX-2, using PacBio and Illumina technologies and RNA sequencing. Their genomic sequences were assembled to contigs with high continuity and showed significant structural differences. The circular mitochondria genomes of both were complete. These genomes provide high-quality resources for deciphering the genomic basis of rapid evolution and host adaptation, identifying genes for avirulence and other important traits, and studying host-pathogen interactions.
\end{abstract}

\section{Genome Announcement}

Puccinia striiformis is an economically important fungal pathogen, causing devastating stripe (yellow) rust diseases on cereal crops and grasses (Hovmøller et al. 2011). Within $P$. striiformis, there are several formae speciales, among which are $P$. striformis f. sp. tritici and $P$. striiformis f. sp. hordei, highly adapted to wheat and barley, respectively (Chen et al. 1995). Even though few genomes have been available for $P$. striiformis f. sp. tritici, they are mainly sequenced using short-read sequencing technologies and are therefore highly fragmented (Cantu et al. 2011, 2013; Cuomo et al. 2017; Kiran et al. 2017; Schwessinger et al. 2018; Zheng et al. 2013). More genomes of $P$. striiformis f. sp. tritici with diverse origins and virulence profiles are needed for comparative genomics to precisely pinpoint the pathogenicity determinants. Moreover, genomes of other formae speciales of $P$. striiformis (e.g., $P$. striiformis f. sp. hordel) are still lacking, even though they are valuable for studying host adaptation and evolution of formae speciales.

$P$. striiformis $\mathrm{f}$. sp. tritici isolate 93-210 and $P$. striiformis $\mathrm{f}$. sp. hordei isolate 93TX-2 were collected in the United States from Montana and Texas, respectively, in 1993. Their formae

\footnotetext{
${ }^{\dagger}$ Corresponding author: Xianming Chen; E-mail: xianming.chen@ars.usda.gov
}

Mention of trade names or commercial products in this publication is solely for the purpose of providing specific information and does not imply recommendation or endorsement by the U. S. Department of Agriculture (USDA). The USDA is an equal opportunity provider and employer.

Accepted for publication 23 May 2018.

(c) 2018 The American Phytopathological Society
Funding

The project was supported by United States Department of Agriculture, Agricultural Research Service (project number 2090-22000-018-00D) and Washington Grain Commission (project number 13C-3061-5665). 
Table 1. Genome statistics of Puccinia striiformis f. sp. tritici isolate $93-210$ and P. striiformis f. sp. hordei isolate $93 \mathrm{TX}-2$

$\begin{array}{lcc}\text { Statistics } & \text { Isolate 93-210 } & \text { Isolate 93TX-2 } \\ \text { Assembly } & & 77,368,122 \\ \text { Genome size in contigs (bp) } & 84,627,802 & 44.40 \\ \text { GC content (\%) } & 44.39 & 562 \\ \text { Number of contigs } & 493 & 263 \\ \text { Number of contigs >100 kb } & 243 & 110 \\ \text { Contig N50 } & 83 & 218,468 \\ \text { Contig N50 length (bp) } & 295,440 & 137,666 \\ \text { Mean contig size (bp) } & 171,659 & 15,976 \\ \text { Annotation } & 16,513 & 93.00 \\ \text { Protein-coding genes } & 95.10 & 626 \\ \text { Annotation completeness (\%) } & 648 & 26.41 \\ \text { Transfer RNA genes } & 30.46 & 34.2 \\ \text { Transposable elements (Mb) } & 36.03 & 9.43 \\ \text { Transposable elements (\%) } & 11.79 & 9.14 \\ \text { Class I (retrotransposons) (Mb) } & 10.22 & 7.84 \\ \text { Class II (DNA transposons) (Mb) } & 8.45 & \\ \text { Others }{ }^{\mathrm{a}} \text { (Mb) } & \mathrm{Mb} \text { (Mb) }\end{array}$

a Others include sequences of satellites, simple repeats, and low complexity.

speciales status was confirmed by pathogenicity tests of both isolates on both sets of wheat and barley cultivars used to differentiate races of $P$. striiformis $\mathrm{f}$. sp. tritici and $P$. striiformis f. sp. hordei, respectively. Genomic DNA was extracted as previously described (Xia et al. 2017). A DNA library with fragment sizes of approximately 500 bp was prepared and was sequenced using the Illumina HiSeq PE150 technology. Meanwhile, a PacBio RS II library was constructed for both isolates and was sequenced using P4-C2 chemistry with 16 singlemolecule real-time cells, generating 5.55- and 6.27-Gb reads for $P$. striiformis $\mathrm{f}$. sp. tritici (93210 ) and $P$. striiformis f. sp. hordei (93TX-2), respectively, about $70 \times$ genome coverage. The Illumina reads were trimmed, corrected, and filtered. In total, 23,750,223 pairs, with a mean read length of $148 \mathrm{bp}$ and comprising 7,030,066,008 bp, passed all tested criteria and were used in subsequent analyses for $P$. striiformis f. sp. tritici (93-210), about 87× genome coverage. Similarly, a total of $20,801,841$ pairs, with a mean read length of $141 \mathrm{bp}$ and comprising 5,880,525,786 bp, were used in subsequent analyses for $P$. striiformis $f$. sp. hordei (93TX-2), about 70x genome coverage. The high-quality and error-corrected Illumina reads were fed to program Proovread v2.14.0 (Hackl et al. 2014) to correct the potential sequencing errors in the PacBio long reads. Assembler Falcon (with Falcon-unzip) (Chin et al. 2016) was used to de novo assemble the corrected PacBio reads for the nuclear genome, and Canu v1.4 (Koren et al. 2017) was used to assemble the mitochondrial genome. The intermediate assemblies were optimized by program Metassembler v1.5 (Wences and Schatz 2015). The assemblies were finalized and were manually corrected after polishing, using the paired-end Illumina reads and software Pilon (version 1.2.2) (Walker et al. 2014). To improve the accuracy of genome annotation, we sequenced mitochondrial RNA for both isolates. Total RNA was extracted from fresh urediniospores, germinated urediniospores, and infected wheat leaves 2 and 7 days after inoculation. Fresh urediniospores, germinated urediniospores, and infected leaves were ground separately in a mortar in liquid nitrogen. Total RNA was extracted from frozen powder using the Trizol reagent (Thermo Fisher Scientific), following the manufacturer's instruction. RNA quality was checked using the Agilent2100 Bioanalyzer (Agilent Technologies). The RNA samples from different stages of each isolate were mixed into one sample at a 1:1:1:1 ratio for RNA sequencing. The preparation of RNA-Seq library and Illumina HiSeq PE150 sequencing were performed at the Novogen Corporation Inc. Trimmed and corrected RNA-Seq reads were aligned to the reference genome using HISAT2 version 2.1.0 (Kim et al. 2015). An automated genome annotation pipeline MAKER version 3.00 (Holt \& Yandell, 2011) was used for gene model calling. The a. initio gene prediction was performed using AUGUSTUS, SNAP, and GeneMark programs (Korf 2004; Stanke et al. 2006). Repeats were masked using RepeatModeler (Institute for Systems Biology). We also provided $P$. striiformis $\mathrm{f}$. sp. tritici expressed sequence tag and protein sequences as external evidence for gene-model predictions. All predicted gene models and inferred $5^{\prime}$ and $3^{\prime}$ untranslated regions were integrated to produce final gene models. In total, three iterative 
runs of MAKER were performed to produce the final gene set for both $P$. striiformis $\mathrm{f}$. $\mathrm{sp}$. tritici and $P$. striiformis $\mathrm{f}$. sp. hordei assemblies. The annotation completeness was evaluated using BUSCO v2.0.1 (Simão et al. 2015). More detailed procedures on genome assembly and annotation are available upon request, since the space is limited in this announcement.

A total of 84 and $77 \mathrm{Mb}$ were assembled into 493 and 562 contigs for $P$. striiformis $\mathrm{f}$. sp. tritici (93-210) and $P$. striiformis f. sp. hordei (93TX-2), respectively (Table 1), which were much better in continuity than most previously available $P$. striiformis $\mathrm{f}$. $\mathrm{sp}$. tritici genomes (Cantu et al. 2011, 2013; Cuomo et al. 2017; Kiran et al. 2017; Zheng et al. 2013) and in high quality comparable to a recently assembled $P$. striiformis f. sp. tritici genome of isolate 104E 137A, also using the PacBio technology (Schwessinger et al. 2018). The assemblies presented here are one haplotype of the dikaryotic genome. We predicted 16,513 and 15,976 protein-coding genes in $P$. striiformis f. sp. tritici (93-210) and $P$. striiformis $\mathrm{f}$. sp. hordei (93TX2), respectively (Table 1). Among them, 46.26 and $45.68 \%$ were validated by the RNA sequences of these isolates obtained in this study. The annotations of the two genomes have high completeness, at $95.10 \%$ for $P$. striiformis f. sp. tritici $(93-210)$ and $93.00 \%$ for $P$. striiformis $\mathrm{f}$. sp. hordei (93TX-2). In addition to nuclear genomes, we also generated the complete mitochondrial (mt) genomes of $P$. striiformis $\mathrm{f}$. sp. tritici (93-210) and $P$. striiformis f. sp. hordei (93TX-2). The complete mt genomes of both isolates were circular and $101 \mathrm{~kb}$ in length with a low GC content (31\%).

Since isolates $P$. striiformis f. sp. tritici (93-210) and $P$. striiformis $\mathrm{f}$. sp. hordei (93TX-2) represent two formae speciales in $P$. striiformis that are highly adapted to different hosts, the genome sequences we present here provide unique resources for studying genomics of host adaptation of devastating plant pathogens in agriculture.

The sequence data have been deposited in the National Center for Biotechnology Information (NCBI) database with BioProject accession PRJNA422914, sample accessions SAMN08200485 for $P$. striiformis f. sp. tritici (93-210) and SAMN0800486 for $P$. striiformis f. sp. hordei (93TX-2). The assembled and annotated genome sequences have been deposited in NCBI with accessions PKSL00000000 for $P$. striiformis f. sp. tritici (93-210) and PKSM00000000 for $P$. striiformis f. sp. hordei (93TX-2).

\section{Acknowledgment}

The Chinese Scholarship Council scholarship to C. Xia is highly appreciated. We thank the Kamiak high performance computing clusters in Washington State University for providing computing resources.

\section{Literature Cited}

Cantu, D., Govindarajulu, M., Kozik, A., Wang, M., Chen, X., Kojima, K. K., Jurka, J., Michelmore, R. W., and Dubcovsky, J. 2011. Next generation sequencing provides rapid access to the genome of Puccinia striiformisf. sp. tritici, the causal agent of wheat stripe rust. PLoS One 6:e24230.

Cantu, D., Segovia, V., MacLean, D., Bayles, R., Chen, X., Kamoun, S., Dubcovsky, J., Saunders, D. G. O., and Uauy, C. 2013. Genome analyses of the wheat yellow (stripe) rust pathogen Puccinia strifformis f. sp. tritici reveal polymorphic and haustorial expressed secreted proteins as candidate effectors. BMC Genomics 14:270.

Chen, X., Line, R. F., and Leung, H. 1995. Virulence and polymorphic DNA relationships of Puccinia striformis $\mathrm{f}$. sp. hordei to other rusts. Phytopathology 85:1335-1342.

Chin, C.-S., Peluso, P., Sedlazeck, F. J., Nattestad, M., Concepcion, G. T., Clum, A., Dunn, C., O'Malley, R., Figueroa-Balderas, R., Morales-Cruz, A., Cramer, G. R., Delledonne, M., Luo, C., Ecker, J. R., Cantu, D., Rank, D. R., and Schatz, M. C. 2016. Phased diploid genome assembly with single-molecule real-time sequencing. Nat. Methods 13:1050-1054.

Cuomo, C. A., Bakkeren, G., Khalil, H. B., Panwar, V., Joly, D., Linning, R., Sakthikumar, S., Song, X., Adiconis, X., Fan, L., Goldberg, J. M., Levin, J. Z., Young, S., Zeng, Q., Anikster, Y., Bruce, M., Wang, M., Yin, C., McCallum, B., Szabo, L. J., Hulbert, S., Chen, X., and Fellers, J. P. 2017. Comparative analysis highlights variable genome content of wheat rusts and divergence of the mating loci. G3Genes Genom. Genet. 7:361-376.
Hackl, T., Hedrich, R., Schultz, J., and Förster, F. 2014. proovread: Large-scale highaccuracy PacBio correction through iterative short read consensus. Bioinformatics 30:3004-3011.

Holt, C., and Yandell, M. 2011. MAKER2: An annotation pipeline and genomedatabase management tool for second-generation genome projects. BMC Bioinformatics 12:491.

Hovmøller, M. S., Sørensen, C. K., Walter, S., and Justesen, A. F. 2011. Diversity of Puccinia striiformis on cereals and grasses. Annu. Rev. Phytopathol. 49: 197-217.

Kim, D., Langmead, B., and Salzberg, S. L. 2015. HISAT: A fast spliced aligner with low memory requirements. Nat. Methods 12:357-360.

Kiran, K., Rawal, H. C., Dubey, H., Jaswal, R., Bhardwaj, S. C., Prasad, P., Pal, D., Devanna, B. N., and Sharma, T. R. 2017. Dissection of genomic features and variations of three pathotypes of Puccinia striiformis through whole genome sequencing. Sci. Rep. 7:42419.

Koren, S., Walenz, B. P., Berlin, K., Miller, J. R., Bergman, N. H., and Phillippy, A. M. 2017. Canu: Scalable and accurate long-read assembly via adaptive $k$-mer weighting and repeat separation. Genome Res. 27:722-736.

Korf, I. 2004. Gene finding in novel genomes. BMC Bioinformatics 5:59.

Schwessinger, B., Sperschneider, J., Cuddy, W. S., Garnica, D. P., Miller, M. E., Taylor, J. M., Dodds, P. N., Figueroa, M., Park, R. F., and Rathjen, J. P. 2018. A near complete haplotype-phased genome of the dikaryotic wheat stripe rust 
fungus Puccinia striiformis f. sp. tritici reveals high interhaplotype diversity. MBio 9:e02275-17.

Simão, F. A., Waterhouse, R. M., Ioannidis, P., Kriventseva, E. V., and Zdobnov, E. M. 2015. BUSCO: Assessing genome assembly and annotation completeness with single-copy orthologs. Bioinformatics 31:3210-3212.

Stanke, M., Schöffmann, O., Morgenstern, B., and Waack, S. 2006. Gene prediction in eukaryotes with a generalized hidden Markov model that uses hints from external sources. BMC Bioinformatics 7:62.

Walker, B. J., Abeel, T., Shea, T., Priest, M., Abouelliel, A., Sakthikumar, S., Cuomo, C. A., Zeng, Q., Wortman, J., Young, S. K., and Earl, A. M. 2014. Pilon: An integrated tool for comprehensive microbial variant detection and genome assembly improvement. PLoS One 9:e112963.
Wences, A. H., and Schatz, M. C. 2015. Metassembler: Merging and optimizing de novo genome assemblies. Genome Biol. 16:207.

Xia, C., Wang, M., Cornejo, O. E., Jiwan, D. A., See, D. R., and Chen, X. 2017. Secretome characterization and correlation analysis reveal putative pathogenicity mechanisms and identify candidate avirulence genes in the wheat stripe rust fungus Puccinia striiformis $\mathrm{f}$. sp. tritici. Front. Microbiol. 8:2394.

Zheng, W., Huang, L., Huang, J., Wang, X., Chen, X., Zhao, J., Guo, J., Zhuang, H., Qiu, C., Liu, J., Liu, H., Huang, X., Pei, G., Zhan, G., Tang, C., Cheng, Y., Liu, M., Zhang, J., Zhao, Z., Zhang, S., Han, Q., Han, D., Zhang, H., Zhao, J., Gao, X., Wang, J., Ni, P., Dong, W., Yang, L., Yang, H., Xu, J.-R., Zhang, G., and Kang, Z. 2013. High genome heterozygosity and endemic genetic recombination in the wheat stripe rust fungus. Nat. Commun. 4:2673. 